\title{
What's happening in covid-19 ICUs? An intensive care doctor answers some common questions
}

Alison Pittard, dean of the Faculty of Intensive Care Medicine, answers Abi Rimmer's queries-and four intensivists tell Emma Wilkinson how they're coping

\author{
Abi Rimmer ${ }^{1}$, Emma Wilkinson freelance journalist ${ }^{2}$
}

${ }^{1}$ The BMJ; ${ }^{2}$ Sheffield, UK

\section{How has it been possible to significantly increase the number of intensive care beds?}

Intensive care can't be provided anywhere except in the intensive care unit (ICU) unless it's purely on a temporary basis. In order to increase capacity during the current pandemic we have had to look at other areas of the hospital where we could safely provide this high level of care. This has led to the postponement of non-urgent surgery to liberate space, staff, and equipment. Anaesthetists have many skills that are transferable to support the care of the critically ill. We've also developed a rapid induction programme, with the Royal College of Surgeons, to help train other specialists to help. There has been a dramatic change in the way we normally work, necessitating a move away from 1:1 nurse:patient ratios.

\section{What are the risks of this move away from $1: 1$ ratios?}

There are concerns about lowering our normal standard of care, not only in terms of patient safety but also the impact on staff of having to work in environments and in ways that they don't normally. The current covid-19 staffing guidance outlining a team approach stretches the critical care team but is supplemented by non-critical care staff as a means of maintaining safety. Any further dilution may compromise this safety and could have a negative impact on staff health and wellbeing.

It is the ratio of staff to patient that's the biggest change to the normal standard of care; it means we're not delivering the same standard of intensive care as we'd like to. At the current time we can provide the treatments we would do normally, but a doctor may not examine the patient as frequently, for example; response time to an alarm may be slower.
Is building ventilators going to help if you don't have the staff with the skills and experience to manage ventilated patients, even with the move to lower nursing ratios?

We've tried to change the way that we work so that there's a healthcare worker at every bed space and they'll be supervised by an intensive care nurse, but on a 1:6 rather than 1:1 ratio. This has been made possible by looking at generic tasks that can be done by someone who is not an intensive care nurse, thereby freeing them up to do more "intensive care" for more patients. At some point the nurse will not be physically able to manage any increase in workload and therefore, yes, it will not matter how many ventilators we have.

\section{Why are people being ventilated on their fronts?}

This is called prone ventilation and is a recognised treatment for acute respiratory distress syndrome (ARDS), which occurs in people who are worst affected by covid-19. The lungs must get oxygen into the bloodstream and remove carbon dioxide at the same time. In ARDS this is impaired, and by moving people on to their front it improves the blood flow through the parts of the lung that are less damaged, making it easier to get oxygen in and carbon dioxide out.

How do you prioritise which patients should be de-escalated from ICU and do you feel we have enough capacity in 


\section{interim, post-ICU rehabilitation beds before people transition back to wards?}

We reduce the level of care patients receive as their underlying condition improves. This is very variable and is related to what was initially wrong with them, how long they have been in ICU, and if there have been any complications during their stay. It's a very individual thing and isn't done on a prioritisation basis. As the patient improves and no longer requires the highest level of care, they frequently still need more care and nursing than can be provided in a normal ward environment, therefore they remain in the ICU. A lack of what we would call enhanced care services leads to longer lengths of stay for the patient on ICU and potentially could have an impact on our ability to admit another patient who needs ICU.

\section{Is there a problem with morale, distress, or burnout among ICU staff, and how could we support your staff better to deal with it?}

ICU staff are highly trained and are used to dealing with uncertainty, however the current situation is unprecedented and there is a considerable emotional, physical, and psychological burden on them. Under normal circumstances, ICU mortality for all conditions is $20 \%$ : that means $80 \%$ of people coming to ICU survive. Currently, in cases of covid-19, the ICU mortality is between $40 \%$ and $60 \%$ so this places a bigger emotional burden on staff, especially as a covid-19 positive person may not be able to have visitors if their family is also symptomatic or isolating. Staff are having to do more communication with families and carers over the phone, which is not what they are used to.

ICU is a high quality service and staff take pride in delivering the best quality, person centred care they can. The current pandemic requires staff to take extra care for their safety using personal protective equipment (PPE) while doing highly complex procedures and care for patients, at the same time ensuring they don't get infected themselves and can stay healthy. Wearing PPE is difficult and staff generally can't wear it for more than two hours without needing a break. Communication wearing PPE is also more difficult and much of ICU care and treatment requires people to work together as teams.

Supporting staff better at present means other people in the hospital helping on ICU where they are able and the public following government advice to stay at home as this puts less pressure on ICUs and staff.

\section{Could we do anything to improve links between ICU and palliative care so that when people are extubated or de-escalated we could do more to provide a dignified and peaceful death?}

ICU and palliative care can work together to help people understand what an ICU stay means for them and consider their advance care planning. The NICE guidance for covid-19 emphasises the importance of considering do not attempt resuscitation orders and advance care plans as well as end of life care when reviewing the whole patient pathway. The Faculty of Intensive Care Medicine has worked with the Royal College of Physicians to produce a resources toolkit to support that guidance, at www.criticalcarenice.org.uk, which includes specific sections on end of life care.

\section{Is there anything that other hospital clinicians could do to help your work and is there anything you wish they understood better?}

NICE guidance emphasises the role of all clinicians involved in patient care before ICU consideration. It is important that opportunities are taken, before a person deteriorates, to discuss their wishes and for clinicians who know the patient well to work with them to determine their care options. ICU clinicians are expert decision makers and are the experts in determining critical care treatments and its likelihood of achieving what a person wants, but other clinicians have a significant role in determining whether a referral to critical care is appropriate.

\section{How are intensive care doctors coping?}

How will we deal with survivors?

The disease is not behaving like any viral pneumonia l've seen before and I'm having to manage 10 ventilated patients at once. The patients are severely ill despite many of them previously being fit and well. Our usual "tricks" don't seem to work, and we've started to change our management using information from centres that have already treated more patients. There's a lot of pride in the NHS and the way it has responded, but we're only at the beginning of a long haul of difficult and challenging work.

How we deal with the survivors is something l've started to think about in the past few days; and how do we maintain morale over the next few weeks when we see so many recently healthy people dying?

Peter Hampshire, clinical lead for critical care, Royal Liverpool Hospital

Difficult decisions with little information

We have expanded from 46 to more than 70 beds with the potential to grow to 260 . Early planning has been key and there's been a lot of cross-specialty collaboration with this novel and unusual disease.

One of the hardest parts for clinicians is having to confront families with news of admission, deterioration, and death, sometimes in a very short space of time, over a phone. Some patients have presented very late, severely hypoxic with multiorgan failure leaving critical care staff having to make difficult decisions with little information. We will soon face the question of "is it over?" as admissions slow, but this will change again if restrictions are lifted, bringing the return of many more conventional critical care admissions to be managed alongside covid-19.

Gary Mills, consultant in anaesthesia and intensive care medicine, Sheffield Teaching Hospitals Foundation Trust

We are working well but risk fatigue

We have cohorted all covid-19 positive patients into our main ICU and non-covid patients are being looked after in a satellite ICU. This means we're operating a second tier of consultant cover, which works well but runs the risk of increasing fatigue. The PPE makes working in ICU more tiring physically and mentally. Medically, we are well staffed and we've had several doctors in training return to us. They have seen exams, recruitment, and progression all suspended and yet have come to work every day full of enthusiasm and good humour. I'm genuinely humbled and uplifted by their spirit.

Rosaleen Baruah, consultant in critical care and anaesthesia, Western General Hospital, Edinburgh

\section{Our biggest concern is communication with relatives}

We had reasonable warning this was coming and started our surge plan early. It's like nothing we've ever seen-the patients are desperately unwell and their length of stay is prolonged. Our biggest concern is communication with relatives-something we usually pride ourselves on. For them not to visit and us not to see them face to face is very hard. I sat with a patient who died the other night, did all I could to make them feel they weren't alone but this is such a departure from normality and it definitely shook me on an emotional level.

Morale is good; there's a siege mentality and the team have pulled together. I just hope we can weather the storm.

David Hepburn, intensive care consultant, Royal Gwent Hospital, Newport

Competing interests: We have read and understood BMJ policy on declaration of interests and have no relevant interests to declare. 
Published by the BMJ Publishing Group Limited. For permission to use (where not already granted under a licence) please go to http://group.bmj.com/group/rights-licensing/

permissions 